\title{
Inventaire des espèces de mouches des fruits sur goyave dans la région de Yaoundé au Cameroun
}

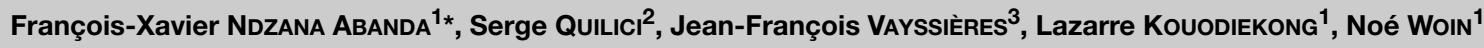

\author{
${ }^{1}$ Irad, Programme Fruits, BP \\ 2067, IRAD Nkolbisson, \\ Messa-Yaoundé, Cameroun \\ ndzanaabanda@yahoo.fr \\ 2 Cirad, UMR PVBMT, Cirad / \\ Université de la Réunion, Pôle \\ de Protection des Plantes, \\ 7 chemin de I'IRAT, 97410, \\ Saint-Pierre, île de la Réunion, \\ France \\ serge.quilici@cirad.fr \\ ${ }^{3}$ Cirad, UPR Production \\ fruitière, IITA, Biological \\ Control Center for Africa, 08 \\ BP 0932, Tri postal, Cotonou, \\ Bénin \\ j.vayssieres@cgiar.org
}

* Correspondance et tirés à part

Reçu le 29 mai 2007 Accepté le 1 octobre 2007

Fruits, 2008, vol. 63, p. 19-26 (C) 2008 Cirad/EDP Sciences All rights reserved DOI: $10.1051 /$ fruits:2007041 www.fruits-journal.org RESUMEN ESPAÑOL, p. 26

\section{Inventory of fruit fly species on guava in the area of Yaounde, Cameroon.}

Abstract - Introduction. The aim of this study was to identify the main species of fruit flies (Diptera, Tephritidae) of economic importance in guava orchards in the central province of Cameroon. Materials and method. This work was completed in three localities (Yaounde, Bikok and Essé) of the wetland area of Cameroun. A total of 270 infested guava fruits was collected and monitored for emergence of fruit flies. Results. The collected fruits made it possible to collect 1260 puparia, from which three species of fruit flies were identified: Ceratitis anonae, which accounted for $64 \%$ of the adults obtained, Bactrocera invadens (35\%) and B. mesomelas (1\%). The first two species, present in the three localities investigated, are of economic importance. B. invadens is a new invasive species for Cameroon, while B. mesomelas, not observed around Yaounde, has lately been reported in this country. Conclusion. This first inventory will have to be followed by a study on the distribution of these species, their host-plant range, the evolution of their populations and their natural enemies. More specific complementary studies should make it possible to improve knowledge on the species of the fruit flies in Cameroon in order to develop an integrated management of these important pests.

Cameroon / Psidium guajava / fruits / Tephritidae / Ceratitis anonae / Bactrocera invadens / Bactrocera mesomelas / identification

\section{Inventaire des espèces de mouches des fruits sur goyave dans la région de} Yaoundé au Cameroun.

Résumé - Introduction. Cette étude a eu pour objectif d'identifier les principales espèces de mouches des fruits (Diptera, Tephritidae) d'importance économique, inféodées au goyavier dans les vergers fruitiers de la province du Centre, au Cameroun. Matériel et méthode. Ce travail a été réalisé dans trois localités (Yaoundé, Bikok et Essé) de la zone humide du pays. Un total de 270 fruits infestés a été collecté, puis mis en observation jusqu'à émergence des adultes. Résultats. Les fruits collectés ont permis de récolter 1260 puparia à partir desquelles trois espèces de mouches des fruits ont été identifiées : Ceratitis anonae qui représente $64 \%$ des adultes obtenus, Bactrocera invadens (35\%) et B. mesomelas (1\%). Les deux premières espèces, présentes dans les trois localités, sont d'importance économique. $B$. invadens est une nouvelle espèce invasive pour le Cameroun, tandis que $B$. mesomelas, non observée à la périphérie de Yaoundé, est nouvellement citée pour ce pays. Conclusion. Ce premier travail d'inventaire devra être suivi d'une étude sur la distribution des espèces identifiées, la gamme de leurs plantes-hôtes, l'évolution de leurs populations et leurs ennemis naturels. Des études complémentaires plus spécifiques devraient permettre d'améliorer les connaissances sur les espèces de mouches de fruits du Cameroun en vue de la mise en place d'une lutte intégrée contre ces importants ravageurs.

Cameroun / Psidium guajava / fruits / Tephritidae / Ceratitis anonae / Bactrocera invadens / Bactrocera mesomelas / identification 


\section{Introduction}

Le goyavier (Psidium guajava L.), de la famille des myrtacées, est un arbre fruitier que l'on retrouve pratiquement dans toutes les aires géographiques du Cameroun. Il est ainsi cultivé en jardin de case, comme culture associée dans les plantations de cacao (Theobroma cacao L.) et de café (Coffea spp.), ou encore dans les cultures vivrières. Cette espèce fruitière, qui pousse souvent de façon spontanée, est de plus en plus cultivée dans de grands vergers.

Au Cameroun, la production de goyaves est estimée à $6000 \mathrm{t}$ [1]. Elle est vendue en majeure partie pour la consommation locale en frais, mais elle est aussi transformée en jus, confiture ou pulpe. La goyave est également utilisée comme source de remèdes pour soigner certains maux et elle peut servir occasionnellement à l'alimentation des animaux.

En zone forestière humide au sud du territoire, la production de goyaves s'étend sur deux saisons de culture : la grande saison va d'août à novembre et la petite d'avril à mai [2]. La pluviométrie moyenne annuelle de cette zone est de $1400 \mathrm{~mm}$ et la goyave est un fruit présent pratiquement toute l'année sur le marché.

Dans le cadre de la diversification des productions agricoles, ce fruit constitue une importante source de revenus pour les planteurs comme pour les revendeurs concernés. Jadis considéré comme une culture peu exigeante, le goyavier doit faire face depuis quelques temps à de fortes contraintes de production parmi lesquelles figurent les problèmes phytosanitaires et de nombreux ravageurs. Les mouches des fruits (Tephritidae) en sont, à l'heure actuelle, les plus sérieux.

Les mouches des fruits ont une grande importance économique, car elles causent aux fruits et légumes charnus des dommages sérieux, nuisant ainsi au commerce tant intérieur qu'international de ces productions [3]. Les contraintes dues aux infestations des mouches des fruits réduisent par exemple de $40 \%$ la production des mangues en Afrique de l'Est [4]. Les femelles pondent leurs œufs, par petits groupes, sous l'épiderme des fruits-hôtes. Les larves se développent dans la pulpe du fruit, puis le quittent en fin de développement larvaire pour se transformer en puparia dans le sol. Les piqûres de ponte des mouches sont susceptibles d'occasionner également une infection secondaire des fruits par des bactéries et des champignons ; il s'ensuit l'éclatement du fruit et la décomposition de la chair.

Il existe peu de publications - ou alors elles sont très anciennes [5, 6] - concernant l'inventaire des Tephritidae d'Afrique centrale, leur biologie ou les méthodes de lutte. Toutefois, un travail récent sur les mouches de fruits du genre Ceratitis et leurs plantes hôtes dans les régions afrotropicales [7] fait état de la présence des espèces suivantes :

- Ceratitis (Pterandrus) anonae Graham sur mangue (Mangifera indica L.), goyave (Psidium guajava L.), avocat (Persea americana Miller), café (Coffea canephora Pierre ex A. Froehner) et mandarine (Citrus reticulata Blanco);

- Ceratitis capitata (Wiedemann) sur café ; - Ceratitis (Ceratalaspis) cosyra Walker sur mangue.

Deux études ponctuelles sur tomate (Lycopersicom esculentum Mill.), qui ont fait l'objet de collectes répétées de fruits, ont été réalisées au Cameroun [8, 9]. Pour l'Afrique de l'Ouest, au contraire, on dispose d'une série de travaux récents [10-16].

En préalable à l'étude de la distribution, au Cameroun, des espèces de mouches des fruits d'importance économique et de leurs ennemis naturels, il nous est paru important de compléter le travail d'inventaire déjà effectué dans ce pays. Notre étude a eu pour premier objectif de recenser les espèces de mouches de fruits inféodées au goyavier dans la région de Yaoundé, puis, dans un second temps, nous avons recherché d'éventuels ennemis naturels de ces ravageurs.

\section{Matériel et méthode}

Les collectes de fruits ont été effectuées par une méthode souvent utilisée $[8,15,17,18]$ qui permet de réaliser des inventaires relativement exhaustifs. 


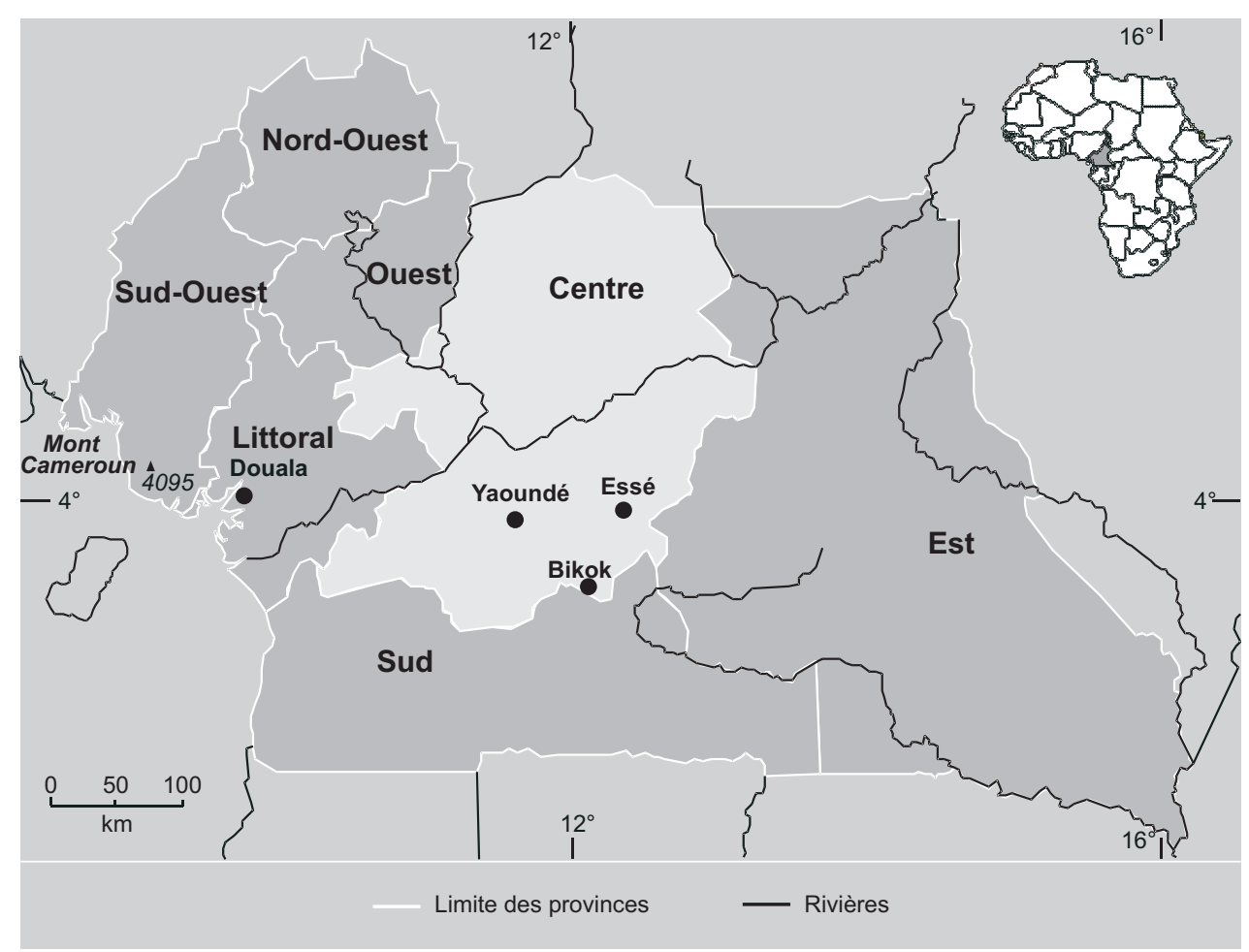

\subsection{Choix et description des localités}

Les goyaves échantillonnées ont été collectées du mois d'août au mois d'octobre 2004 dans trois localités de la province du centre du Cameroun (figure 1) : Bikok, Essé et Yaoundé. Bikok et Essé sont situées de part et d'autre de Yaoundé, la première localité étant à $45 \mathrm{~km}$ au sud-est et la seconde à $70 \mathrm{~km}$ au nord-est de cette ville. Les trois localités ont été choisies du fait de leur proximité et de la disponibilité de goyaviers dans les vergers. La collecte des fruits a eu lieu pendant la grande saison de production de goyaves dans la zone forestière humide du Cameroun.

À Bikok (lat. 3 37' 60” N, long. $11^{\circ} 25^{\prime}$ 60 " E, alt. $712 \mathrm{~m}$ ), nous avons retenu un verger de 4 ha dont le matériel végétal provenait de l'Institut de Recherche Agricole pour le Développement (IRAD, Yaoundé, Cameroun). Cette région, incluse dans la zone forestière humide avec une pluviométrie moyenne annuelle de $1400 \mathrm{~mm}$, compte également beaucoup de vergers paysans.

À Essé (lat. $4^{\circ}$ 4' 60” N, long. 1152' 60” E, alt. $686 \mathrm{~m}$ ), un verger de près de 40 ha a été sélectionné dans une zone de transition entre la zone forestière et la zone de savane. Ce verger est constitué de différentes espèces fruitières : goyavier (Psidium guajava L.), agrume (Citrus spp.), corossolier (Annona muricata L.), safoutier (Dacryodes edulis (G. Don) H.J. Lam), avocatier (Persea americana), manguier (Mangifera indica), papayer (Carica papaya L.), etc.

À Yaoundé (lat. $3^{\circ} 54^{\prime} \mathrm{N}$, long. $11^{\circ} 31^{\prime} \mathrm{E}$, alt. $726 \mathrm{~m}$ ), nous avons choisi le verger expérimental de l'IRAD de Minkoameyos et de sa périphérie. Yaoundé est situé dans une zone forestière humide avec une pluviométrie moyenne annuelle qui varie de (1350 à 1750) $\mathrm{mm}[2]$.

Les trois vergers retenus sont menés en polyculture. Les arbres sont âgés d'une dizaine d'années pour les vergers de Bikok et Essé et d'une vingtaine d'années pour celui de Yaoundé.
Figure 1.

Localisation des sites inventoriés (Bikok, Essé et Yaoundé) au Cameroun pour identifier les mouches de fruits infestant les goyaviers dans la province du Centre. 
Figure 2.

Importance relative de trois espèces de mouches de fruits (Ceratitis anonae, Bactrocera invadens et $B$. mesomelas) inventoriées sur goyaviers dans trois localités du centre du Cameroun.

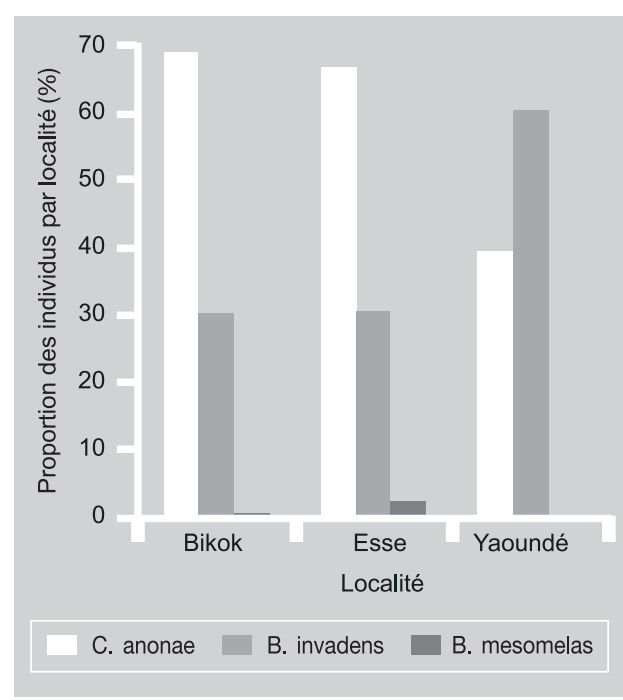

\section{2. Échantillonnage}

Des goyaves présentant des traces visibles d'infestation ont été collectées et rapportées au laboratoire. Trente fruits infestés ont été récoltés par localité à l'occasion de chacune de trois visites sur le terrain effectuées à intervalles de deux semaines. Au laboratoire, les fruits ont été étiquetés pour chaque numéro de visite et chaque localité. Chaque fruit a été pesé, puis mis dans une boîte d'élevage numérotée, préalablement remplie à moitié de sable sec tamisé recouvert par un papier buvard. Ce papier buvard a été remplacé tous les 5 jours afin d'éviter un excès d'eau dans les boîtes.
Chaque boîte a été recouverte d'une fine couche de mousseline transparente et aérée, permettant d'éviter la fuite des adultes de mouches après leur émergence. Chaque semaine, le sable a été tamisé afin de recueillir les puparia. Lorsque le sable était trop humide (à cause de l'eau suintant du fruit), il était remplacé par du sable sec. En fin de développement larvaire, deux à trois semaines après leur isolement, les larves sorties du fruit se sont transformées en puparia dans le sable.

Après tamisage du sable, les puparia recueillis à l'aide de pinces souples ont été placés dans des boîtes de Petri garnies d'un papier buvard légèrement humide, jusqu'à l'émergence suivie tous les 2 jours pour récupération des adultes. Une pré-détermination du genre des mouches a été effectuée sur place.

Les déterminations précises ont été effectuées par le Dr. Marc De Meyer du Musée Royal d'Afrique Centrale de Tervuren (Belgique) pour les Ceratitis et par le Dr. Ian White du Natural History Museum de Londres pour Bactrocera invadens. Les mouches issues de notre élevage ont d'ailleurs constitué des paratypes pour la description de cette nouvelle espèce invasive [19]. Les résultats ont été analysés à l'aide du logiciel de statistique SPSS. D'éventuelles différences significatives entre les trois localités visitées ont été étudiées par analyses de variance, suivies d'une comparaison multiple des moyennes par un test de Student avec correction de Bonferroni (tableau I).

\section{Tableau I.}

Nombre moyen d'adultes de mouches des fruits, émergés par fruit et par site, à partir de la collecte de 90 goyaves infestées, dans trois localités au centre du Cameroun.

\begin{tabular}{lcccc} 
Localité & $\begin{array}{c}\text { Nombre moyen } \\
\text { de mouches par fruit }\end{array}$ & Erreur standard & \multicolumn{2}{c}{ Intervalle de confiance à $95 \%$} \\
\hline Esse & $6,72 \mathrm{a}$ & 0,71 & 5,32 & Borne inférieure \\
Bikok & $5,13 \mathrm{a}$ & 0,71 & 3,73 & Borne supérieure \\
Yaoundé & $2,14 \mathrm{~b}$ & 0,71 & 0,74 & 6,13 \\
\hline
\end{tabular}

$a, b$ : Les nombres affectés de la même lettre dans une même colonne ne sont pas significativement différents (ANOVA, $P<0,01$ ). 


\section{Tableau II.}

Nombre moyen de mouches des fruits, obtenues par kg de goyaves, à partir de la collecte de 90 goyaves infestées, dans trois localités au centre du Cameroun.

\begin{tabular}{|c|c|c|c|c|}
\hline \multirow[t]{2}{*}{ Localité } & \multirow[t]{2}{*}{ Nombre de mouches } & \multirow[t]{2}{*}{ Erreur standard } & \multicolumn{2}{|c|}{ Intervalle de confiance à 95 \% } \\
\hline & & & Borne inférieure & Borne supérieure \\
\hline Bikok & 103,38 a & 10,28 & 83,13 & 123,63 \\
\hline Esse & $56,80 \mathrm{~b}$ & 10,28 & 36,55 & 77,05 \\
\hline Yaoundé & $46,30 \mathrm{~b}$ & 10,28 & 26,05 & 66,55 \\
\hline
\end{tabular}

$a, b$ : Les nombres affectés de la même lettre dans une même colonne ne sont pas significativement différents (ANOVA, $P<0,01$ ).

\section{Tableau III.}

Taux d'infestation par la mouche des fruits, mesuré à partir de l'observation de 90 goyaves collectées dans trois localités du centre du Cameroun.

\begin{tabular}{lcccc} 
Localité & $\begin{array}{c}\text { Nombre } \\
\text { de fruits infestés }\end{array}$ & $\begin{array}{c}\text { Poids moyen d'un fruit } \\
(\mathrm{g})\end{array}$ & $\begin{array}{c}\text { Poids total des fruits } \\
(\mathrm{kg})\end{array}$ & $\begin{array}{c}\text { Taux d'infestation' } \\
(\%)\end{array}$ \\
\hline Bikok & 72 & 59,5 & 5,3 & 80,0 \\
Esse & 69 & 129,5 & 11,6 & 76,7 \\
Yaoundé & 57 & 55,2 & 5,0 & 63,3 \\
&
\end{tabular}

\section{Résultats et discussion}

\subsection{Les mouches répertoriées : espèces et distribution}

Les goyaves collectées (90 fruits par localité) ont permis de récolter au total 1260 puparia. La localité d'Essé est celle qui a permis de récupérer le plus grand nombre d'adultes (605 soit $48 \%$ du total), suivie par celle de Bikok (462 soit $37 \%$ du total), puis celle de Yaoundé (193 soit $15 \%$ du total).

Les trois espèces de Tephritidae qui ont été identifiées après émergences des adultes appartiennent à deux genres de la sousfamille des Dacinae : le genre Ceratitis (Mac Leay), dans la tribu des Ceratitidini, avec une espèce, Ceratitis (Pterandrus) anonae Graham, et le genre Bactrocera (Macquart), dans la tribu des Dacini, avec deux espèces, Bactrocera (Bactrocera) invadens (Drew, Tsuruta et White) et Bactrocera (Gymnoda- cus) mesomelas (Bezzi). Ceratitis anonae et Bactrocera invadens sont considérées comme des espèces d'importance économique.

Ceratitis anonae a été l'espèce la plus abondante (64\% des 1260 puparia obtenus) ; $B$. invadens a représenté $35 \%$ des puparia et $B$. mesomelas, $1 \%$. Les espèces $C$. anonae et $B$. invadens ont été trouvées dans les trois sites visités, bien qu'en proportions variables, alors que $B$. mesomelas a été absente des fruits collectés à Yaoundé. C. anonae s'est révélée l'espèce la plus abondante à Bikok (69\%) et Esse (67\%), tandis que $B$. invadens a été dominante à Yaoundé (61\%) (figure 2).

L'analyse de variance montre que le nombre moyen de mouches par kg de goyaves infestées a varié de manière significative $(P<0.01)$ en fonction des sites de collecte (tableau II). Les fruits provenant de Bikok ont un nombre moyen de mouches plus 
élevé (103 mouches $\mathrm{kg}^{-1}$ ) que ceux d'Esse ( 57 mouches $\mathrm{kg}^{-1}$ ) ou de Yaoundé ( $46 \mathrm{mou}$ ches $\left.\cdot \mathrm{kg}^{-1}\right)$. La moyenne pour les trois localités a été de 69 mouches·kg-1 de fruits. Dans une étude récemment effectuée au Kenya [18], le nombre moyen de B. invadens et $C$. cosyra sur mangues variait de $(3$ à 97,2) mouches $\cdot \mathrm{kg}^{-1}$ de fruits (858 fruits infestés échantillonnés).

Le taux de fruits infestés a également varié en fonction du site de collecte : de $80 \%$ à Bikok à 63,3\% à Yaoundé (tableau III).

Des émergences de parasitoïdes ont été aussi fréquemment observées. Toutefois, leur identification n'a pas encore été effectuée.

\subsection{Importance économique des espèces de Tephritidae}

\subsubsection{Bactrocera invadens}

Outre le Cameroun, l'espèce $B$. invadens, d'introduction récente, a déjà été signalée dans une quinzaine de pays africains (Bénin, Ghana, Guinée, Guinée équatoriale, Kenya, Mali, Nigéria, Ouganda, République démocratique du Congo, Sénégal, Soudan, Tanzanie, Togo) [16, 18-21]. Par ailleurs, des données non publiées indiquent sa présence au Burkina Faso, en Côte-d'Ivoire, au Niger, en République du Congo et en Sierra Leone (Vayssières, non publ.), ainsi maintenant qu'aux Comores dans l'Océan indien (Quilici et al., non publ.). La zone d'origine de l'espèce se situerait au Sri Lanka et au sud de l'Inde [19]. En Tanzanie, elle est signalée comme une espèce de basse à moyenne altitude [20,21], ce qui correspond à la situation de trois zones d'études visitées lors de nos collectes au Cameroun.

Parmi les principales plantes hôtes cultivées de $B$. invadens, identifiées jusqu'ici, se trouvent le goyavier (Psidium guajava L.), le manguier (Mangifera indica L.), et le corossolier (Annona muricata L.) [16, 18, 20, 21]. Les autres plantes hôtes signalées sont le bibacier ou néflier du Japon (Eriobotryajaponica (Thumb), le pomelo (Citrus paradisi Macfad.), le mandarinier (Citrus reticulata Blanco), l'oranger [Citrus sinensis (L.) Osbeck], l'avocatier (Persea americana Mill.), le papayer (Carica papaya L.), le pêcher (Prunus persica L.), la tomate (LyCO- persicon esculentum Mill.), le bananier (Musa spp.), la pastèque (Citrullus lanatus Thumb.), le concombre (Cucumis sativus L.), le pommier de Cythère (Spondias cytherea Sonn.) et le badamier (Terminalia catappa L.) [16, 18, 20, 21]. D'autres plantes hôtes, cultivées ou sauvages, restent à déterminer.

Il serait intéressant d'effectuer des études similaires sur goyavier dans d'autres zones agro-écologiques du Cameroun afin de compléter l'inventaire des espèces de mouches des fruits inféodées à ce fruit hôte et de préciser la distribution de $B$. invadens dans l'ensemble du pays.

\subsubsection{Ceratitis anonae}

En Afrique, C. anonae est un ravageur important pour une dizaine d'espèces fruitières cultivées ou sauvages [3, 7, 10, 14, 15] parmi lesquelles figure le goyavier. Nous avons obtenu des émergences de $C$. anonae à partir de mangues et de corossols dans les localités de Bikok et Essé durant la période de cette étude. L'espèce est également mentionnée comme un ravageur important du manguier en Guinée [14] et au Mali [15].

Ceratitis anonae est largement répandue sur le continent africain : Côte d'Ivoire, Gabon, Ghana, Guinée Conakry, Guinée équatoriale, Kenya, Mali, Nigéria, Ouganda, République centrafricaine, République démocratique du Congo, République du Congo, Sao Tomé et Principe, Tanzanie et Togo [7, 22]. Hormis le goyavier, les autres plantes hôtes de C. anonae connues sont l'avocatier (Persea americana), le cacao (Theobroma cacao L.), les caféiers arabica (Coffea arabica L.) et robusta (Coffea canephora), ainsi que Psidium cattleyanum Sabine, Citrus spp., Cola cordifolia (Cav.) R. Br, Annona senegalensis Pers., Terminalia catappa, Solanum mauritianum Scop. et Ficus spp.

\subsubsection{Bactrocera mesomelas}

L'espèce B. mesomelas, nouvellement identifiée au Cameroun, n'avait été obtenue à partir de fruits que sur goyavier au Gabon en 1996 (Vayssières, non publ.). Elle a été signalée en Afrique au sud du Sahara dans les pays suivants : Cameroun, Côte d'Ivoire, Gabon, Ghana, Guinée, Mali, Nigeria et République démocratique du Congo [23]. La 
faible abondance de cette espèce que nous avons constatée pourrait être expliquée en partie par la délimitation de notre étude qui a porté sur une seule plante hôte et sur une aire géographique limitée. Il se pourrait également que la période de notre étude n'ait pas coïncidé avec une période d'abondance de cette espèce. Des travaux portant sur une large gamme de plantes hôtes et sur une période de temps plus longue permettraient de mieux appréhender sa distribution au Cameroun. Par ailleurs, une étude récente en Tanzanie mentionne la présence de $B$. (Gymnodacus) amplexa (Munro), une espèce très proche de B. mesomelas [20], toutefois, en très faibles effectifs.

\subsubsection{Les autres espèces observées}

Nous avons également pu noter la présence de C. (Ceratalaspis) cosyra (Walker) sur mangues et corossols collectés à Essé et Bikok, et $C$. (Pardalaspis) punctata (Wiedemann) sur goyaves récupérées dans la périphérie de Yaoundé et à Essé. Il s'est agi alors de récoltes de fruits isolés, gérés selon la même méthode que celle décrite plus haut.

\section{Conclusion}

L'étude préliminaire réalisée au Cameroun nous a permis de recenser trois espèces de mouches de fruits inféodées au goyavier: $C$. anonae, B. invadens et B. mesomelas. Si $C$. anonae avait déjà été signalée dans ce pays [2, 6], B. invadens est une espèce récemment décrite [17] qui n'avait jamais été mentionnée au Cameroun. À la suite de sa récente apparition, il s'avère qu'elle cohabite avec les Ceratitis spp. indigènes du Cameroun.

De futurs travaux devraient nous permettre de préciser la distribution de B. mesomelas et sa gamme de plantes hôtes au Cameroun. De même, des études devront être conduites afin de préciser la distribution et l'écologie de B. invadens dans le pays, cette espèce ayant un impact économique majeur au Cameroun, comme dans les sous-régions d'Afrique centrale et de l'Ouest.

L'étude des espèces de mouches des fruits présentes au Cameroun devrait donc être poursuivie dans d'autres zones agro- écologiques du pays, sur diverses plantes hôtes d'intérêt commercial (manguier, papayer, agrumes, etc.) et sur une plus longue période, afin de compléter nos connaissances sur ces ravageurs et d'évaluer leur importance économique.

\section{Remerciements}

Nous remercions la Fondation Germaine Cousin pour son soutien financier à cette étude, ainsi que les Dr. Marc De Meyer et Lan White pour leurs appuis à l'identification des espèces. Marci à Michel Havard pour la relecture du manuscrit. Nos remerciements vont également aux paysans de Bikok, Essé et Yaoundé qui ont mis gracieusement à notre disposition leurs vergers. Merci à Luc Dubog et Bella Manga pour leur encouragement tout au long de ce travail.

\section{Références}

[1] Temple L., Quantification des productions et des échanges des fruits et légumes au Cameroun, Cah. Agric. 10 (2) (2001) 87-94.

[2] Kuate J., Bella-Manga, Damesse F., Kouedikong L., Ndindeng S.A., David O., Parrot L., Enquêtes sur les cultures fruitières dans les exploitations familiales agricoles en zone humide du Cameroun, Fruits 61 (2006) 373-387.

[3] White I.M., Elson- Harris M.M., Fruit flies of economic significance: their identification and bionomics, CAB Int., London, UK, 1992, $601 \mathrm{p}$.

[4] Lux S.A., African fruit fly initiative: Pan-African initiative to promote productivity and trade of fruits and vegetables through management of African fruit flies, ICIPE, Nairobi, Kenya, 1999, 28 p.

[5] Silvestri F., Report of an expedition to Africa in search of natural enemies of fruit flies (Trypaneidae), Hawaii, Board Agric. For., Div. Entomol, Bull. 3 (1914) 1-176.

[6] Nonveiller G., Catalogue commenté et illustré des insectes du Cameroun d'intérêt agricole, Inst. Prot. Plantes, Mém. XV, Beograd, Serbie,1984, $210 \mathrm{p}$.

[7] De Meyer M., Copeland R.S, Lux S.A., Mansell M, Quilici S., Wharton R, White I.M., Zenz N.J., Liste commentée des plantes hôtes des mouches des fruits (Diptera: Tephritidae) afrotropicales du genre Ceratitis, MRAC, Doc. Zool. Vol. 27, Tervuren, Belgique, 2002. 
[8] Tindo M., Tamo M., La mouche des fruits Dacus punctatifrons (Diptera: Tephritidae) comme problème de production de la tomate dans la région de la Lékié (Sud Cameroun), Ann. Soc. Entomol. Fr. (N.S.) 35 (suppl.) (1999) 525-527.

[9] Okolle J., Ntonifor N.N., Field ovipositional behavior and laboratory studies on development of Dacus punctatifrons (Diptera: Tephritidae) on tomato, Insect Sci. 12 (2005) 393-398.

[10] N'Guetta K., Inventaire des insectes de fruits récoltés dans le Nord Côte d'Ivoire, Fruits 49 (1994) 502-503.

[11] Vayssières J.F., Sangaré M., Enquête sur le potentiel de production de mangues en Haute Guinée, Projet FAC/IRAG, Minist. Dév. Rural, Conakry, Guinée, 1995, 18 p.

[12] Barbet A., Suivi des populations de cératites (Diptera: Tephritidae) en Nord Côte d'Ivoire dans la perspective d'une lutte raisonnée, CNEARC, Mém., Montpellier, France, 2000, $52 \mathrm{p}$.

[13] Noussourou M., Diarra B., Lutte intégrée contre les mouches des fruits, Sahel IPM 6 (1995) 2-13.

[14] Vayssières J.F., Kalabane S., Inventory and fluctuations of the catches of Diptera Tephritidae associated with mangoes in Coastal Guinea, Fruits 55 (2000) 259-270.

[15] Vayssières J.F., Sanago F., Noussourou M., Inventory of the fruit fly species (Diptera: Tephritidae) linked to the mango tree in Mali and tests of integrated control, Fruits 62 (2007) 329-341.
[16] Vayssières J.F., Georgen G., Lokossou O., Dossa P., Akponon C., A new Bactrocera species in Benin among mango fruit fly (Diptera: Tephritidae) species, Fruits 60 (2005) 371-377.

[17] Sales F., Les mouches des fruits dans les territoires de Nouvelle-Calédonie et de Walliset-Futuna, Fruits 53 (1998) 41-46.

[18] Ekesi S., Nderitu P.W., Rwomushana I., Field infestation, life history and demographic parameters of the fruit fly Bactrocera invadens (Diptera: Tephritidae) in Africa, Bull. Entomol. Res. 96 (4) 2006 379-386.

[19] Drew R.A.I, Tsuruta K., White I.M., A new species of pest fruit fly (Diptera: Tephritidae: Dacinae) from Sri Lanka and Africa, Afr. Entomol. 13 (1) 2005 149-154.

[20] Mwatawala M.W., De Meyer M., Makundi R.H., Maerere A.P., Biodiversity of fruit flies (Diptera: Tephritidae) at orchards in different agro-ecological zones of the Morogoro region, Tanzania, Fruits 61 (2006) 321-332.

[21] Mwatawala M.W., De Meyer M., Makundi R.H., Maerere A.P., Seasonality and host utilization of the invasive fruit fly, Bactrocera invadens (Diptera: Tephritidae) in central Tanzania, J. Appl. Entomol. 130 (9-10) (2006) 530-537.

[22] De Meyer M., Freidberg A., Revision of the subgenus Ceratitis (Pterandrus) Bezzi (Diptera: Tephritidae), in: Freidberg A. (Ed.), Biotaxonomy of Tephritoidea, Isr. J. Entomol. 35/36 (2006) 197-315.

[23] White I.M., Taxonomy of the Dacina (Diptera: Tephritidae) of Africa and the middle East, Afr. Entomol. Mem. 2 (2006) 1-156.

Inventario de las especies de moscas de la fruta en la guayaba, en la región de Yaundé en Camerún.

Resumen - Introducción. Este estudio tuvo como objetivo la identificación de las principales especies de moscas de la fruta (Diptera, Tephritidae) de importancia económica hospedada en el guayabo en los vergeles fruteros de la provincia del Centro, en Camerún. Material y método. Se realizó este trabajo en tres localidades (Yaundé, Bikok y Essé) de la zona húmeda del país. Se colectaron un total de 270 frutos infestados, que se pusieron después bajo observación hasta emergencia de los adultos. Resultados. Los frutos colectados permitieron cosechar 1260 puparia a partir de los cuales se identificaron tres especies de moscas de la fruta: Ceratitis anonae, la cual representa el $64 \%$ de los adultos obtenidos; Bactrocera invadens (35\%) y B. mesomelas $(1 \%)$. Las dos primeras especies, presentes en las tres localidades poseen una importancia económica. B. invadens es una nueva especie invasiva en Camerún, mientras que B. mesomelas, no observada en la periferia de Yaundé, se cita nuevamente en este país. Conclusión. Este primer trabajo de inventario deberá proseguirse con un estudio sobre la distribución de las especies identificadas, la gama de sus plantas- huésped, la evolución de sus poblaciones y sobre sus enemigos naturales. Unos estudios complementarios más específicos deberían permitir la mejora de los conocimientos sobre las especies de moscas de la fruta de Camerún en vistas de la instauración de una lucha integrada contra esta plaga importante.

Camerún / Psidium guajava / frutas / Tephritidae / Ceratitis anonae / Bactrocera invadens / Bactrocera mesomelas / identificación 